\title{
Quantum Fisher Information in Two-Qubit Pure States
}

\author{
Wan-Fang Liu • Li-Hua Zhang • Chun-Jie Li
}

Received: 4 March 2010 / Accepted: 9 July 2010 / Published online: 28 July 2010

(C) The Author(s) 2010. This article is published with open access at Springerlink.com

\begin{abstract}
In terms of quantum Fisher information (QFI), a quantity $\chi^{2}$ was introduced by Pezzé and Smerzi (Phys. Rev. Lett. 102 100401, 2009). They pointed out that the inequality $\chi^{2}<1$ was a sufficient condition for multiparticle entanglement. For the two-qubit symmetric states, we found that the inequality $\chi^{2}<1$ is a necessary and sufficient condition for entanglement and spin squeezing, and that $\chi^{2}$ is equal to the second kind of spin squeezing parameter $\xi_{2}^{2}$. For the two-qubit asymmetric states, it is only a sufficient condition. In order to make it a necessary and sufficient condition, we extend the concept of the QFI and $\chi^{2}$, and generalize the relations among the entanglement measurement, the spin squeezing parameters and $\chi^{2}$ in symmetric pure states to arbitrary pure states.
\end{abstract}

Keywords Quantum Fisher information · Criterion · Two-qubit system · Pure state

\section{Introduction}

Quite recently, in terms of quantum Fisher information, Pezzé and Smerzi [1] introduced a sufficient criterion for multiparticle entanglement

$$
\chi^{2}=N / F_{Q}\left[\rho_{\text {in }}, S_{\vec{n}}\right]<1,
$$

where $N$ is the number of particles of the system under consideration, and $F_{Q}$ is the QFI [2-13]. Thus the quantity $F_{Q} / N$ gives the mean quantum Fisher information per particle, which is reciprocal to the quantity $\chi^{2}$. In addition, $\rho_{\text {in }}$ represents the input state, $S_{\vec{n}}$ is the collective operator defined as $S_{\vec{n}} \equiv S \cdot \vec{n}=\sum_{l=1}^{N} \sigma_{l}$, where $\sigma_{l}$ is a Pauli matrix operator on the $l$ th particle, and $\vec{n}$ denotes the direction along which $\chi^{2}$ is detected.

W.-F. Liu $(\bowtie) \cdot$ L.-H. Zhang

School of Physics and Electric Engineering, Anqing Teachers College, Anqing 246011, China

e-mail: wanfangliu2008@gmail.com

C.-J. Li

College of Physics, Changchun Normal University, Changchun 130032, China 
The QFI is closely connected to the phase estimation in metrology and quantum sensors [6]. Generally, the output state is a linear rotation of the input state by an angle $\varphi: \rho_{\text {out }}=$ $e^{i \varphi S_{\vec{n}}} \rho_{\mathrm{in}} e^{-i \varphi S_{\vec{n}}}$. The estimation of the angle $\varphi$ is bounded by the shot-noise limit $\Delta \varphi_{\mathrm{SN}} \equiv$ $1 / \sqrt{N}$. According to the quantum Cramer-Rao theorem [2, 3], the phase sensitivity $\Delta \varphi$ has a lower bound limit

$$
\Delta \varphi_{\mathrm{QCR}}=1 / \sqrt{F_{Q}\left[\rho_{\mathrm{in}}, S_{\vec{n}}\right]}=\chi / \sqrt{N} .
$$

If $\chi^{2}<1$, the state is entangled, and is useful for sub-shot-noise sensitivity of phase estimation. Especially, when $\chi=1 / \sqrt{N}$, the estimation sensitivity can reach the Heisenberg limit $1 / N$. From (2), we see that the smaller $\chi$ (or bigger $F_{Q}$ ) is, the better the phase estimation of the input state is.

On the other hand, both the QFI and the spin squeezing are connected to the spin fluctuation and the entanglement [14-20,22]. The spin squeezing refers to the minimum spin fluctuation of the plane perpendicular to the mean spin direction, and for pure states, the maximal QFI refers to the maximal spin fluctuation over all directions. These suggest that the QFI has some unknown relations with the spin squeezing. So far, a great deal of efforts have been devoted to the study of the relations between the spin squeezing and the entanglement. It was proposed that the spin squeezing can be used as an indicator for many-particle entanglement [23]. For a two-qubit symmetric state, the spin squeezing is equivalent to its bipartite entanglement [24, 25]. For an arbitrary symmetric multiqubit state, the spin squeezing implies pairwise entanglement, for some special state, the spin squeezing is equivalent to the pairwise entanglement [26]. Recently, the spin squeezing sudden death and the entanglement sudden death were studied in different decoherence channels [27]. However, the study of the relations between the QFI and the entanglement or between the QFI and the spin squeezing is lacking. Therefore, one issue naturally arises: are there quantitative relations among the quantity $\chi^{2}$, the spin squeezing and the entanglement for two-qubit system? In this paper, we verify that they are mutually equivalent, and $\chi^{2}$ equals to the second kind of spin squeezing parameter for pure states of two-qubit systems.

Our work is organized as follows. In Sect. 2, we give an introduction to the QFI, $\chi^{2}$ and two spin squeezing parameters. Then in Sect. 3, we study the QFI and $\chi^{2}$ for symmetric pure states of two-qubit, for example, we numerically study $\chi^{2}$ and the first spin squeezing parameter in two nonlinear models with three arbitrary traverse fields. In Sect. 4, we first extend the concept of the QFI and the quantity $\chi^{2}$, then study the QFI and $\chi^{2}$ for arbitrary two-qubit pure states. Finally, a conclusion is given in Sect. 5.

\section{Quantum Fisher Information and Spin Squeezing}

The QFI, which lies at the heart of the quantum estimation theory, is the information we know about a certain parameter in a quantum state. It is related to the degree of statistical distinguishability of a quantum state from its neighbors in parameter space $[4,5]$. Considering a quantum state $\rho(\varphi)$ with $\varphi$ a parameter, the QFI for $\varphi$ is defined as

$$
F_{Q}(\varphi)=\operatorname{tr}\left[\rho(\varphi) L_{\varphi}^{2}\right]
$$

where $L_{\varphi}$, the so-called symmetric logarithmic derivative, is determined by the following equation $[2,3]$

$$
\partial_{\varphi} \rho(\varphi)=\frac{1}{2}\left[L_{\varphi} \rho(\varphi)+\rho(\varphi) L_{\varphi}\right],
$$


where $\partial_{\varphi}:=\partial / \partial \varphi$, and $L_{\varphi}$ can be resolved by rewriting the above equation under the eigenbasises of $\rho$. Then the QFI can be obtained as

$$
F_{Q}(\varphi)=\sum_{i} \frac{\left(\partial_{\varphi} p_{i}\right)^{2}}{p_{i}}+2 \sum_{i \neq j} \frac{\left(p_{i}-p_{j}\right)^{2}}{p_{i}+p_{j}}\left|\left\langle\varphi_{j} \mid \partial_{\varphi} \varphi_{i}\right\rangle\right|^{2},
$$

where $\left|\varphi_{i}\right\rangle$ and $p_{i}$ are eigenvectors and eigenvalues of $\rho$, respectively. If we want to estimate $\varphi$ through measuring $\rho(\varphi)$, the variance of $\varphi$ is limited by a fundamental bound, which is given by the quantum Cramer-Rao (QCR) $[2,3]$ inequality

$$
\Delta \varphi \geq \Delta \varphi_{\mathrm{QCR}} \equiv \frac{1}{\sqrt{F_{Q}(\varphi)}} .
$$

If $F_{Q}(\varphi)$ is large, the variance of our estimation is small.

To connect the QFI with our problem, we consider the following scenario, which arises in general quantum metrology process. Firstly, we prepare a system consisting of $N$ spin-1/2 particles and this system plays a role of probe. Then it will be rotated around direction $\vec{n}$ for a certain angle $\varphi$. This process (quantum channel) is formulated as $\rho(\varphi)=e^{i \varphi S_{\vec{n}}} \rho_{\text {in }} e^{-i \varphi S_{\vec{n}}}$. Finally, we estimate the angle $\varphi$ by measuring $\rho(\varphi)$. The variance of our estimation is bounded by the QCR. The QFI for $\varphi$ is denoted as $F_{Q}\left[\rho_{\text {in }}, S_{\vec{n}}\right]$. As the eigenvalues of $\rho(\varphi)$ and $\rho_{\text {in }}$ are the same, the expression of QFI (1) is just $[1,7,28]$

$$
F_{Q}\left[\rho_{\text {in }}, S_{\vec{n}}\right]=2 \sum_{i, j} \frac{\left(p_{i}-p_{j}\right)^{2}}{p_{i}+p_{j}}\left|\left\langle\psi_{j}\left|S_{\vec{n}}\right| \psi_{i}\right\rangle\right|^{2} .
$$

For a pure state $\rho_{\text {in }}=|\psi\rangle\langle\psi|$, the QFI is simplified as

$$
F_{Q}\left[\rho_{\mathrm{in}}, S_{\vec{n}}\right]=4\left(\Delta S_{\vec{n}}\right)^{2}
$$

with $\left(\Delta S_{\vec{n}}\right)^{2} \equiv\left\langle S_{\vec{n}}^{2}\right\rangle-\left\langle S_{\vec{n}}\right\rangle^{2}$. The following relation

$$
\Delta \varphi \geq \frac{\chi}{\sqrt{N}}
$$

gives a connection between $\Delta \varphi$ and $\chi$. If $\chi^{2}=1$, the lower bound of $\Delta \varphi$, i.e. $\Delta \varphi_{\mathrm{QCR}}$ is $1 / \sqrt{N}$, the so-called shot-noise limit. If $\chi^{2}<1$, the state is entangled, so the sub-shot-noise limit is attained, and when $\chi^{2}=1 / N$, the estimation of $\Delta \varphi_{\mathrm{QCR}}$ can reach the Heisenberg limit, $1 / N$, which is in principle the lowest variance for estimation. It's noticed that the above QFI depends on the direction $\vec{n}$, and thus is not definite for a given state. In practice, we hope to find a direction $\vec{n}$, for which the corresponding QFI is maximal, and thus $\chi^{2}$ is minimal. However, except for some special cases, this is not an easy task. There is a nearoptimal approach, with which we can find the mean spin direction $\vec{n}_{1}=\langle\vec{S}\rangle /|\langle\vec{S}\rangle|$, at first, and then the maximal QFI in the plane orthogonal to $\vec{n}_{1}$ can be determined.

This approach is used in computing the spin squeezing parameters as follows. There are two kinds of the spin squeezing parameters given respectively by Kitagawa and Ueda [14], and Wineland [15],

$$
\xi_{1}^{2}=\frac{4 \min \left(\Delta S_{\vec{n}_{\perp}}\right)^{2}}{N}, \quad \xi_{2}^{2}=\frac{N \min \left(\Delta S_{\vec{n}_{\perp}}\right)^{2}}{\left|\left\langle S_{\vec{n}}\right\rangle\right|^{2}},
$$


where subscript $\vec{n}_{\perp}$ refers to an arbitrary axis in the plane $\left(\vec{n}_{2}, \vec{n}_{3}\right)$ which is perpendicular to the mean spin direction $\vec{n}_{1}$, where the three directions were given by [26, 29, 30]

$$
\left(\begin{array}{l}
\vec{n}_{1} \\
\vec{n}_{2} \\
\vec{n}_{3}
\end{array}\right)=\left(\begin{array}{ccc}
\sin \theta \cos \phi & \sin \theta \sin \phi & \cos \theta \\
-\sin \phi & \cos \phi & 0 \\
-\cos \theta \cos \phi & -\cos \theta \sin \phi & \sin \theta
\end{array}\right)\left(\begin{array}{l}
\vec{n}_{x} \\
\vec{n}_{y} \\
\vec{n}_{z}
\end{array}\right) .
$$

The second kind of the spin squeezing parameter is a criteria for multipartite entanglement $[23,26]$, the inequality $\xi_{i}^{2}<1(i=1,2)$ indicates that the state is entangled and spin is squeezed. The explicit expression for $\xi_{1}^{2}$ is obtained in Ref. [26, 29, 30]. If $\left\langle S_{\vec{n}_{1}}\right\rangle \neq 0$, we have

$$
\xi_{1}^{2}=\frac{N}{2}\left[\left\langle S_{\vec{n}_{2}}^{2}+S_{\vec{n}_{3}}^{2}\right\rangle-\left\{\left\langle S_{\vec{n}_{2}}^{2}-S_{\vec{n}_{3}}^{2}\right\rangle^{2}+\left\langle\left[S_{\vec{n}_{2}}, S_{\vec{n}_{3}}\right]_{+}\right\rangle^{2}\right\}^{1 / 2}\right]
$$

where $[A, B]_{+}=A B+B A$ denotes the anti-commutator. Furthermore, if $\left\langle\left[S_{\vec{n}_{2}}, S_{\vec{n}_{3}}\right]_{+}\right\rangle=0$, which means there are no correlations between $S_{\vec{n}_{2}}$ and $S_{\vec{n}_{3}}$, we have

$$
\xi_{1}^{2}=\frac{4 \min \left(\left\langle S_{\vec{n}_{2}}^{2}\right\rangle,\left\langle S_{\vec{n}_{3}}^{2}\right\rangle\right)}{N} .
$$

Considering the state has symmetry under any permutation, the second kind of spin squeezing parameter can be reduced to

$$
\xi_{2}^{2}=\frac{\xi_{1}^{2}}{\left\langle\sigma_{\vec{n} 1}\right\rangle^{2}} .
$$

To obtain the maximal QFI, i.e., the maximal variance of $S_{\vec{n}}$, we follow similar steps as above. If $\left[S_{\vec{n}_{1}}, S_{\perp}\right]_{+}=0$, which means $S_{\vec{n}_{1}}$ and $S_{\perp}$ has no correlations under state $\rho$, we have [31]

$$
\max \left(\Delta S_{\vec{n}}^{2}\right)=\max \left(\Delta S_{\vec{n}_{1}}^{2}, \max \left(\Delta S_{\perp}\right)^{2}\right),
$$

where

$$
\max \left(\Delta S_{\perp}\right)^{2}=\frac{1}{2}\left[\left\langle S_{\vec{n}_{2}}^{2}+S_{\vec{n}_{3}}^{2}\right\rangle+\left\{\left\langle S_{\vec{n}_{2}}^{2}-S_{\vec{n}_{3}}^{2}\right\rangle^{2}+\left\langle\left[S_{\vec{n}_{2}}, S_{\vec{n}_{3}}\right]_{+}\right\rangle^{2}\right\}^{1 / 2}\right] .
$$

Then the minimal $\chi^{2}$ is

$$
\chi^{2}=\frac{N}{4 \max \left(\Delta S_{\vec{n}_{1}}^{2}, \max \left(\Delta S_{\perp}\right)^{2}\right)} .
$$

Fortunately, in the $X Y$ and the LMG model [22, 32-35], $\vec{n}_{1}$ is along the $z$ axis, and $\left[S_{\alpha}, S_{\beta}\right]_{+}=0, \alpha, \beta \in\{x, y, z\}, \alpha \neq \beta$. Thus $\chi^{2}$ and $\xi_{1}^{2}$ are determined by just computing $\Delta S_{\alpha}$.

\section{Quantum Fisher Information in Two-Qubit Pure States}

3.1 Quantum Fisher Information in the Two-Qubit Symmetric Pure States

Having introduced the QFI and the criterion $\chi^{2}<1$ for the entanglement in Sect. 2, we study the QFI and $\chi^{2}$ in states of pair qubits in this section. We restrict states of pair qubits 
to the triplet sub-space. The collective angular momentum operator belonging to it can be expressed as follows

$$
S_{\mu}=\sum_{i=1}^{2} S_{i, \mu}, \quad \mu \in\{x, y, z\},
$$

where $S_{i, \mu}$ are the spin operators for the $i$ th qubit, and the cyclic commutation relations satisfy

$$
\begin{aligned}
{\left[S_{i, \mu}, S_{i, \nu}\right] } & =i \in_{\mu \nu \gamma} S_{i, \gamma}, \\
{\left[S_{\mu}, S_{\nu}\right] } & =i \in_{\mu \nu \gamma} S_{\gamma}, \quad \mu, \nu, \gamma \in\{x, y, z\} .
\end{aligned}
$$

Considering that the output state is a linear rotation of the input state by an angle $\varphi$, we can treat $\varphi$ as a parameter, then our major arm is to find the maximal spin fluctuation in calculation of the maximal QFI in quantum states.

Lemma 1 For the symmetric state

$$
|\psi\rangle=\sin \alpha|11\rangle+\cos \alpha|00\rangle
$$

there exist the relations among $\chi^{2}, \xi_{1}^{2}, \xi_{2}^{2}$ and $C$

$$
\chi^{2}=\xi_{2}^{2}=\frac{1}{1+C}=\frac{1}{2-\xi_{1}^{2}},
$$

where $|0\rangle$ and $|1\rangle$ are the eigenstates of the spin operator $S_{i, z}$ with the eigenvalue $1 / 2$ and $-1 / 2$, respectively.

Proof For the entangled state

$$
|\psi\rangle=\sin \alpha|11\rangle+\cos \alpha|00\rangle
$$

one can readily verify that the relation among the concurrence $[36,37]$ and two spin squeezing parameters [38]

$$
C=|\sin 2 \alpha|, \quad \xi_{1}^{2}=1-C, \quad \xi_{2}^{2}=\frac{1}{1+C} .
$$

The mean spin direction $\vec{n}_{1}$ is along the $z$-axis and its collective angular momentum operator satisfies the condition

$$
\left\langle\left[S_{z}, S_{x}\right]_{+}\right\rangle=\left\langle\left[S_{z}, S_{y}\right]_{+}\right\rangle=0,
$$

then the maximal spin fluctuation has the following form

$$
\max \left(\Delta S_{\vec{n}}\right)^{2}=\max \left(\Delta S_{z}^{2}, \max \Delta S^{2}(x, y)\right) .
$$

It is easy to calculate the fluctuations $\Delta S_{z}^{2}$ and $\Delta S^{2}(x, y)$. Exactly, we can obtain the fluctuation in the $z$ direction

$$
\Delta S_{z}^{2}=\sin ^{2}(2 \alpha)
$$


Considering the mean spin direction is along the $z$-axis, we can use the method in Refs. [26, $29,30]$ to get the maximum fluctuation in the plane $(x, y)$,

$$
\max \Delta S^{2}(x, y)=\frac{1}{2}\left[\left\langle S_{x}^{2}+S_{y}^{2}\right\rangle+\left|\left\langle S_{+}^{2}\right\rangle\right|\right]=\frac{1+|\sin 2 \alpha|}{2} .
$$

From the two equations above, it readily follows that $\max \Delta S^{2}(x, y)$ is just the maximal spin fluctuation over the whole coordinate space. Finally, the maximal QFI and the minimum $\chi^{2}$ can be reduced to a simple form, namely,

$$
F_{Q}=2(1+|\sin 2 \alpha|), \quad \chi^{2}=\frac{1}{1+|\sin 2 \alpha|} .
$$

Combined with (24), we may easily get the desired result,

$$
\chi^{2}=\xi_{2}^{2}=\frac{1}{1+C}=\frac{1}{2-\xi_{1}^{2}} .
$$

This result is useful to the later lemma.

Lemma 2 For an arbitrary symmetric state of two-qubit

$$
|\tilde{\psi}\rangle=\sin \alpha|\tilde{1} \tilde{1}\rangle+\cos \alpha|\tilde{0} \tilde{0}\rangle
$$

the relations given by (30) are still valid, where states $|\tilde{0}\rangle$ and $|\tilde{1}\rangle$ are eigenstates of the operator $\vec{\sigma}_{i} \cdot n$ with an arbitrary direction $n=(\sin \theta \cos \phi, \sin \theta \sin \phi, \cos \theta)$

$$
\begin{aligned}
& |\tilde{0}\rangle=e^{\frac{-i \phi}{2}} \cos \frac{\theta}{2}|0\rangle+e^{\frac{i \phi}{2}} \sin \frac{\theta}{2}|1\rangle, \\
& |\tilde{1}\rangle=e^{\frac{-i \phi}{2}} \sin \frac{\theta}{2}|0\rangle-e^{\frac{i \phi}{2}} \cos \frac{\theta}{2}|1\rangle
\end{aligned}
$$

with eigenvalue 1 and -1 , respectively.

Proof We begin with the eigenstates $|0\rangle$ and $|1\rangle$ of $\sigma_{i, z}$. Firstly, we perform a rotation of states around the $y$ axis. In terms of the local operations, this rotation can be achieved by unitary transformation $U\left(S_{i, y}\right)=e^{-i \theta S_{i, y}}$. Then, the two eigenstates become

$$
\begin{aligned}
& \left|0^{\prime}\right\rangle=\cos \frac{\theta}{2}|0\rangle+\sin \frac{\theta}{2}|1\rangle, \\
& \left|1^{\prime}\right\rangle=\sin \frac{\theta}{2}|0\rangle-\cos \frac{\theta}{2}|1\rangle .
\end{aligned}
$$

Next, according to a similar procedure, if we perform a rotation of states $\left|0^{\prime}\right\rangle$ and $\left|1^{\prime}\right\rangle$ around the $z$ axis, i.e., unitary transformation $U\left(S_{i, z}\right)=e^{-i \phi S_{i, z}}$, then the states $\left|0^{\prime}\right\rangle$ and $\left|1^{\prime}\right\rangle$ will be transformed to the states $|\tilde{0}\rangle$ and $|\tilde{1}\rangle$ given by (32) and (33). It should be noted that operators $S_{i, \mu}$ act only on the states belonging to its own Hilbert space. Therefore, after performing the collective unitary transformation

$$
U=e^{-i \phi S_{z}} e^{-i \theta S_{y}}
$$


on the state in (23), we can transform the state $|\psi\rangle$ to the state

$$
|\tilde{\psi}\rangle=\cos \alpha|\tilde{0} \tilde{0}\rangle+\sin \alpha|\tilde{1} \tilde{1}\rangle
$$

Now we perform the rotations of the local and collective operators. Under the rotations, we easily obtain the new local and global operators

$$
\tilde{S}_{\mu}=\sum_{i=1}^{2} \tilde{S}_{i, \mu}, \quad \mu \in\{x, y, z\}
$$

with

$$
\tilde{S}_{i, \mu}=U_{i} S_{i, \mu} U_{i}^{+}, \quad \mu \in\{x, y, z\},
$$

where $U_{i}^{+}=e^{i \theta S_{i, y}} e^{i \phi S_{i, z}}$. It is evident that the cyclic commutation relations satisfy

$$
\begin{aligned}
{\left[\tilde{S}_{i, \mu}, \tilde{S}_{i, \nu}\right] } & =i \in_{\mu \nu \gamma} \tilde{S}_{i, \gamma}, \\
{\left[\tilde{S}_{\mu}, \tilde{S}_{\nu}\right] } & =i \in_{\mu \nu \gamma} \tilde{S}_{\gamma}, \quad \mu, \nu, \gamma \in\{x, y, z\}
\end{aligned}
$$

For the all equations referred to the expectation values in Lemma 1, after a simply replacing $S$ by $\tilde{S}$, they are still valid. Therefore, Lemma 1 is invariant under the above rotation. In other words, we need only to perform a corresponding transformation on the collective operators when the state $|\psi\rangle$ is transformed to the state $|\tilde{\psi}\rangle$.

Proposition 1 For an arbitrary symmetric state of pair qubits

$$
|\psi\rangle=a_{1}|00\rangle+a_{2} / \sqrt{2}(|01\rangle+|10\rangle)+a_{3}|11\rangle,
$$

where $a_{1}, a_{2}$ and $a_{3}$ are arbitrary constants, one can find that $\chi^{2}<1$ implies the entanglement and the spin squeezing and vice versa. There exist the relations among them

$$
\chi^{2}=\xi_{2}^{2}=\frac{1}{1+C}=\frac{1}{2-\xi_{1}^{2}}
$$

Proof In view of the exact expression of states $|\tilde{0}\rangle$ and $|\tilde{1}\rangle$, the symmetric state $|\tilde{\psi}\rangle$ can be reduced to a simple form, namely,

$$
|\tilde{\psi}\rangle=A_{1}|00\rangle+A_{2} / \sqrt{2}(|01\rangle+|10\rangle)+A_{3}|11\rangle
$$

with coefficients

$$
\begin{aligned}
& A_{1}=e^{-i \phi}\left(\cos \alpha \cos ^{2} \frac{\theta}{2}+\sin \alpha \sin ^{2} \frac{\theta}{2}\right), \\
& A_{2}=\sqrt{2} \cos \frac{\theta}{2} \sin \frac{\theta}{2}(\cos \alpha-\sin \alpha), \\
& A_{3}=e^{i \phi}\left(\cos \alpha \sin ^{2} \frac{\theta}{2}+\sin \alpha \cos ^{2} \frac{\theta}{2}\right) .
\end{aligned}
$$

By appropriate choice of $\alpha, \theta$ and $\phi$, one can achieve that $A_{1}, A_{2}$ and $A_{3}$ are equal to $a_{1}, a_{2}$ and $a_{3}$, respectively. From Lemma 2, we immediately have Proposition 1. 


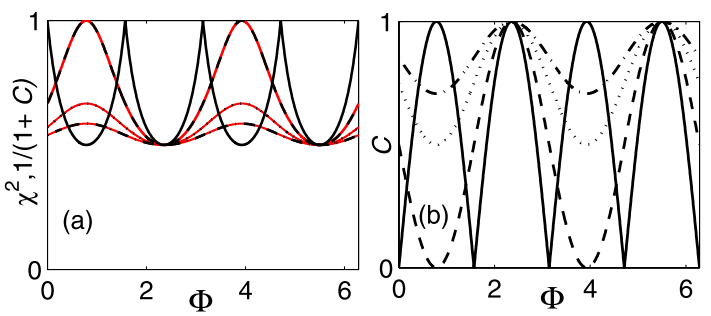

Fig. 1 (Color online) $\chi^{2}, 1 /(1+C)$ and $C$ versus $\Phi$ for different parameter $\Theta$. Parameter takes $\Theta=\pi / 8$ (dashed dotted line), $\Theta=\pi / 6$ (dotted line), $\Theta=\pi / 4$ (dashed line) and $\Theta=\pi / 2$ (solid line), respectively. In $(\mathbf{a}), \chi^{2}$ and $1 /(1+C)$ are presented by black lines and red lines, respectively. In $(\mathbf{b}), C$ was plotted

This proposition shows that for a two-qubit symmetric pure state, the criterion $\chi^{2}<1$, the spin squeezing and the entanglement are equivalent to each other. Moreover, the quantity $\chi^{2}$ equals to the second kind of spin squeezing parameter. In order to illustrate further Proposition 1, we examine a symmetric state including real coefficients

$$
a_{1}=\sin \Theta \cos \Phi, \quad a_{2}=\cos \Theta, \quad a_{3}=\sin \Theta \sin \Phi .
$$

As a function of $\Phi$, we numerically calculate $\chi^{2}, 1 /(1+C)$ and $C$ for the different value of $\Theta$, as shown in Fig. 1(a) and (b). We see that the red lines and black lines were completely overlapping together. These numerical results directly display relations between them presented by Proposition 1, as we expected.

To generate the spin squeezing and the QFI, one-axis twisting model and two-axis model are studied widely $[14,16-21]$. As a natural supplement, we check $\xi_{1}^{2}, 1 /\left(2-\xi_{1}^{2}\right)$ and $\chi^{2}$ of two-qubit system in the one-axis twisting model with three arbitrary external transverse fields

$$
H=2 \kappa S_{x}^{2}+\Omega_{x} S_{x}+\Omega_{y} S_{y}+\Omega_{z} S_{z}
$$

and the two-axis counter twisting model with three arbitrary external transverse fields

$$
H=\kappa\left(S_{x} S_{y}+S_{y} S_{x}\right)+\Omega_{x} S_{x}+\Omega_{y} S_{y}+\Omega_{z} S_{z},
$$

where $\Omega_{i}, i \in\{x, y, z\}$ are the external transverse field strengthes. The initial state of the two-qubit system is $|11\rangle$. For convenience, here, we take $\kappa=1$. For example, we randomly make three strengthes $\Omega_{x}=3, \Omega_{y}=2, \Omega_{z}=1$. In general, the two models above cannot be solved analytically, while numerical calculation can be carried out. We see that there exists the superposition of red line corresponding to $1 /\left(2-\xi_{1}^{2}\right)$ and green line corresponding to $\chi^{2}$, as shown in Fig. 2(a) and (b). Also, these numerical results directly verify the relations between $\chi^{2}$ and $\xi_{1}^{2}$ in Proposition 1 .

\subsection{Quantum Fisher Information in Two-Qubit Asymmetric States}

From the analysis of the previous section, it is known that the criterion $\chi^{2}<1$ for the entanglement works well in symmetric states. However, for asymmetric states, how is the ability of $\chi^{2}<1$ to detect the entanglement? Here, we first present a simple example. Considering a state of two-qubit given by

$$
|\psi\rangle=\sin \alpha|10\rangle+e^{i \beta} \cos \alpha|01\rangle
$$




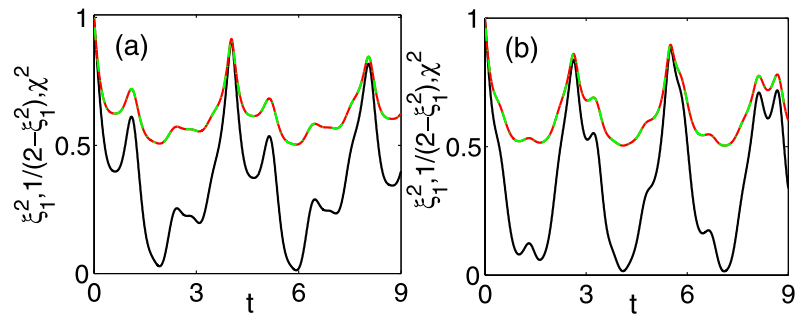

Fig. 2 (Color online) $\xi_{1}^{2}$ (black line), 1/(2- $\xi_{1}^{2}$ ) (red line) and $\chi^{2}$ (green line) versus $t$. (a) and (b) correspond to the one-axis twisting model with three arbitrary external transverse fields and the two-axis twisting model with three arbitrary external transverse fields, respectively

with pairwise entanglement $C=|\sin 2 \alpha|$, it has the property $\left\langle S_{\mu}\right\rangle=0(\mu \in\{x, y, z\})$ and $\left[S_{z}, S_{x}\right]_{+}=\left[S_{z}, S_{y}\right]_{+}=0$, hence the mean spin equals to zero and its direction may be in any direction. Through the same procedure given by the previous section, the maximal spin fluctuation can be obtained as

$$
\max \left(\Delta S_{\vec{n}}\right)^{2}=\frac{1+\cos \beta \sin 2 \alpha}{2} .
$$

Substituting the above equation into (1) and (8) leads to

$$
\begin{aligned}
& F_{Q}= \begin{cases}2(1+\cos \beta C), & \text { if } \sin 2 \alpha>0, \\
2(1-\cos \beta C), & \text { if } \sin 2 \alpha<0,\end{cases} \\
& \chi^{2}= \begin{cases}\frac{1}{1+\cos \beta C}, & \text { if } \sin 2 \alpha>0, \\
\frac{1}{1-\cos \beta C}, & \text { if } \sin 2 \alpha<0 .\end{cases}
\end{aligned}
$$

It is clear that if $\cos \beta=0$, the quantity $\chi^{2}$ always equals to one, and a direct consequence is that $\chi^{2}$ cannot detect whether the above state is an entangled state or not. However, the state in (49) is entangled for $\alpha \neq n \pi / 2$. This fact shows that the criterion $\chi^{2}<1$ implies the entanglement only is a sufficient condition, but not a necessary one. In order to generalize the results of Proposition 1 to arbitrary pure states of two qubits, we will introduce in following section a generalized $\chi_{g}^{2}$ which satisfies the relation given by the proposition above.

\section{Quantum Fisher Information with Local Unitary Invariance}

\subsection{Generalized QFI and New Quantity $\chi_{g}^{2}$}

Having studied the QFI in the symmetric pure states of two-qubit systems, now we explore the possibility of extending the concept of $\chi^{2}$ to arbitrary pure state of two-qubit in order to overcome the problem of (49). Here individual qubits are controllable in the sense that they are accessible to local operations. Firstly, we redefine the total mean spin direction of system with $N$ particles in terms of the local mean spin direction given by [38].

$$
\hat{n}_{i 0}=\frac{\left\langle\vec{\sigma}_{i}\right\rangle}{\left|\left\langle\vec{\sigma}_{i}\right\rangle\right|}, \quad\left|\left\langle\vec{\sigma}_{i}\right\rangle\right|=\left(\left\langle\vec{\sigma}_{i}\right\rangle \cdot\left\langle\vec{\sigma}_{i}\right\rangle\right)^{1 / 2}, \quad i \in N
$$


Considering a mutually orthogonal set $\left(\hat{n}_{i \perp}, \hat{n}_{i \vdash}, \hat{n}_{i 0}\right)$ of unit vector associated with each qubit, we can redefine a collective spin operator in terms of $\vec{\sigma}_{i}$ and $\hat{n}_{i 0}$

$$
J_{0}=\frac{1}{2} \sum_{i=1}^{N} \vec{\sigma}_{i} \cdot \hat{n}_{i 0} .
$$

Then, we can get another collective spin operators perpendicular to $\tilde{J}_{0}$

$$
J_{\perp}=\frac{1}{2} \sum_{i=1}^{N} \vec{\sigma}_{i} \cdot \hat{n}_{i \perp}, \quad J_{\vdash}=\frac{1}{2} \sum_{i=1}^{N} \vec{\sigma}_{i} \cdot \hat{n}_{i \vdash},
$$

which are governed by the cyclic commutation relations. For example, we can easily verify

$$
\left[J_{\perp}, J_{\vdash}\right]=i J_{0} .
$$

Based on this, it leads to the uncertainty relation

$$
\left(\Delta J_{\perp}\right)\left(\Delta J_{\vdash}\right) \geq \frac{1}{2}\left|\left\langle J_{0}\right\rangle\right| .
$$

Analogous to the QFI and the definition of $\chi^{2}$, we define a new QFI with the maximal spin fluctuation $\max \left(\Delta J_{\vec{n}}\right)^{2}$ and a quantity $\chi_{g}^{2}$

$$
F_{g Q}=4 \max \left(\Delta J_{\vec{n}}\right)^{2}, \quad \chi_{g}^{2} \equiv \frac{N}{F_{g Q}} .
$$

We can give a similar sufficient criterion based on the quantity $\chi_{g}^{2}$ for particle entanglement

$$
\chi_{g}^{2}<1 .
$$

Also, the generalized two spin squeezing parameters are given by [38]

$$
\xi_{g 1}^{2}=\frac{4 \min \left(\Delta J_{\perp 0}\right)^{2}}{N}, \quad \xi_{g 2}^{2}=\frac{N \min \left(\Delta J_{\perp 0}\right)^{2}}{\left\langle J_{0}\right\rangle^{2}},
$$

where $\min \left(\Delta J_{\perp 0}\right)^{2}$ is the minimum spin fluctuation in the plane $(\perp, \vdash)$.

We will demonstrate that the condition $\chi_{g}^{2}<1$, the entanglement and the spin squeezing are mutually equivalent for two-qubit pure asymmetric state.

\subsection{Generalized QFI and the Quantity $\chi_{g}^{2}$ in Two-Qubit Asymmetric States}

Now we turn to the study of $\chi_{g}^{2}$ of two-qubit asymmetric pure states. We start from the following lemma.

Lemma 3 For the asymmetric state

$$
|\psi\rangle=\sin \alpha|10\rangle+e^{i \beta} \cos \alpha|01\rangle,
$$

there exist the relations among $\chi_{g}^{2}, \xi_{1}^{2}, \xi_{2}^{2}$ and $C$

$$
\chi_{g}^{2}=\xi_{g 2}^{2}=\frac{1}{1+C}=\frac{1}{2-\xi_{g 1}^{2}} .
$$


Proof We start form the classical state represented by (49)

$$
|\psi\rangle=\sin \alpha|10\rangle+e^{i \beta} \cos \alpha|01\rangle .
$$

It is obvious that

$$
\left|\left\langle\sigma_{i, x}\right\rangle\right|=\left|\left\langle\sigma_{i, y}\right\rangle\right|=0, \quad\left|\left\langle\sigma_{i, z}\right\rangle\right|=|\cos 2 \alpha| .
$$

In view of the definition $\hat{n}_{i 0}$, we can get three collective operators as

$$
\begin{aligned}
& J_{0}=\frac{\cos 2 \alpha\left(\sigma_{1, z}-\sigma_{2, z}\right)}{2|\cos 2 \alpha|}, \\
& J_{\perp}=\frac{\cos 2 \alpha\left(\sigma_{1, x}-\sigma_{2, x}\right)}{2|\cos 2 \alpha|}, \\
& J_{\vdash}=\frac{\left(\sigma_{1, y}+\sigma_{2, y}\right)}{2} .
\end{aligned}
$$

It should be noted that under the new definition of the collective operators, the condition $\left[J_{0}, J_{\perp}\right]_{+}=\left[J_{0}, J_{\vdash}\right]_{+}=0$ is satisfied. Therefore, we have similar results for the fluctuations

$$
\begin{aligned}
\min \left(\Delta J_{\perp 0}\right)^{2} & =\frac{1}{2}\left[\left\langle J_{\perp}^{2}+J_{\vdash}^{2}\right\rangle-\sqrt{\left\langle J_{\perp}^{2}-J_{\vdash}^{2}\right\rangle^{2}+\left\langle\left[J_{\perp}, J_{\vdash}\right]_{+}\right\rangle^{2}}\right], \\
\max \left(\Delta J_{\perp 0}\right)^{2} & =\frac{1}{2}\left[\left\langle J_{\perp}^{2}+J_{\vdash}^{2}\right\rangle+\sqrt{\left\langle J_{\perp}^{2}-J_{\vdash}^{2}\right\rangle^{2}+\left\langle\left[J_{\perp}, J_{\vdash}\right]_{+}\right\rangle^{2}}\right], \\
\max \left(\Delta J_{\vec{n}}\right)^{2} & =\max \left(\Delta J_{0}^{2}, \max \left(\Delta J_{\perp 0}\right)^{2}\right),
\end{aligned}
$$

where $\min \left(\Delta J_{\perp 0}\right)^{2}$ and $\max \left(\Delta J_{\perp 0}\right)^{2}$ were the minimum and the maximal spin fluctuation in the plane perpendicular to $J_{0}$, respectively. By a direct calculation, we can obtain their exact expressions as

$$
\begin{aligned}
\left(\Delta J_{0}\right)^{2} & =\sin ^{2}(2 \alpha), \\
\min \left(\Delta J_{\perp 0}\right)^{2} & =\frac{1-|\sin 2 \alpha|}{2}, \\
\max \left(\Delta J_{\perp 0}\right)^{2} & =\frac{1+|\sin 2 \alpha|}{2} .
\end{aligned}
$$

Furthermore, we have

$$
F_{g Q}=2(1+|\sin 2 \alpha|), \quad \chi_{g}^{2}=\frac{1}{1+|\sin 2 \alpha|} .
$$

Also, we can obtain the expectation value and the second kind of spin squeezing parameter

$$
\left\langle J_{0}\right\rangle^{2}=\cos ^{2}(2 \alpha), \quad \xi_{g 2}^{2}=\frac{1}{1+|\sin 2 \alpha|} .
$$

Then, we have the relations

$$
\chi_{g}^{2}=\xi_{g 2}^{2}=\frac{1}{1+C}=\frac{1}{2-\xi_{g 1}^{2}} .
$$


Based on this lemma we have the following lemma.

Lemma 4 For an arbitrary asymmetric state of two-qubit

$$
|\tilde{\psi}\rangle=\sin \alpha|\tilde{1} \tilde{0}\rangle+e^{i \phi} \cos \alpha|\tilde{0} \tilde{1}\rangle,
$$

there exist the relations among $\chi_{g}^{2}, \xi_{g 1}^{2}, \xi_{g 2}^{2}$ and $C$

$$
\chi_{g}^{2}=\xi_{g 2}^{2}=\frac{1}{1+C}=\frac{1}{2-\xi_{g 1}^{2}} .
$$

Proof Following similar steps leading to Lemma 2, we can transform the state of (49) to the state of (75). Also, we need to perform the corresponding transformation on the three collective operators redefined in (54) and (55). Then, they will become $\tilde{J}_{0}, \tilde{J}_{\perp}, \tilde{J}_{\vdash}$. It is obvious that after the transformation, the cyclic commutation relations do not change. So the thing we only need to do is to complete a replacement $J_{i}$ by $\tilde{J}_{i}, i \in\{\perp, \vdash, 0\}$. By a similar calculation, we can obtain Lemma 4.

Proposition 2 For an arbitrary state

$$
\left.|\psi\rangle=a_{1}|00\rangle+a_{2}|01\rangle+a_{3}|10\rangle\right)+a_{4}|11\rangle
$$

of pair qubits in the standard basis $\{|00\rangle,|11\rangle,|01\rangle,|10\rangle\}$, where $a_{1}, a_{2}, a_{3}$ and $a_{4}$ are arbitrary constants, we have that $\chi_{g}^{2}<1$ implies the entanglement and the spin squeezing and vice versa. There are the relations among them

$$
\chi_{g}^{2}=\xi_{g 2}^{2}=\frac{1}{1+C}=\frac{1}{2-\xi_{g 1}^{2}} .
$$

Proof Following similar procedures leading to Proposition 1, we substitute the exact expressions of states $|\tilde{0}\rangle$ and $|\tilde{1}\rangle$ into the state $|\tilde{\psi}\rangle$, it can be reduced to the state

$$
\left.|\psi\rangle=A_{1}|00\rangle+A_{2}|01\rangle+A_{3}|10\rangle\right)+A_{4}|11\rangle
$$

with the coefficients

$$
\begin{aligned}
& A_{1}=\left(\cos \alpha+e^{-i \beta} \sin \alpha\right) \sin \frac{\theta}{2} \cos \frac{\theta}{2} e^{-i \phi}, \\
& A_{2}=\left(e^{-i \beta} \sin \alpha \sin ^{2} \frac{\theta}{2}-\cos \alpha \cos ^{2} \frac{\theta}{2}\right), \\
& A_{3}=\left(\cos \alpha \sin ^{2} \frac{\theta}{2}-e^{-i \beta} \sin \alpha \cos ^{2} \frac{\theta}{2}\right), \\
& A_{4}=-\left(\cos \alpha+e^{-i \beta} \sin \alpha\right) \sin \frac{\theta}{2} \cos \frac{\theta}{2} e^{-i \phi} .
\end{aligned}
$$

Also, by appropriate of choice of $\alpha, \beta, \theta$ and $\phi$, we get that the coefficients $A_{1}, A_{2}, A_{3}$ and $A_{4}$ are equal to $a_{1}, a_{2}, a_{3}$ and $a_{4}$, respectively. By a direct application of Lemma 4, we can derive Proposition 2. It shows that the results of symmetric pure states can be generalized to arbitrary pure states and reduced to Proposition 1 for symmetric pure states. From this proposition, it is known that $\chi_{g}^{2}$ is local unitary invariant. In other words, states with the same entanglement possess the same QFI and $\chi_{g}^{2}$. 


\section{Conclusions}

In conclusion, we have studied the relations among $\chi^{2}$, the spin squeezing parameters and the pairwise entanglement in two-qubit pure states. For symmetric states, we directly demonstrate that the condition $\chi^{2}<1$, the spin squeezing and the entanglement are equivalent, and $\chi^{2}$ is equal to the second kind of spin parameter $\xi_{2}^{2}$. For arbitrary states, we provide a local unitary invariant version of definition of $\chi^{2}$. In this frame, the relations among the three indicated quantities existing in symmetric states are still satisfied.

Acknowledgements The authors thank helpful discussions with H. N. Xiong, Jian Ma. This work is supported by College Young Talents Foundation of Anhui Province with Grant no. 2010SQRL107, and the Natural Science Foundation of the Education Department of Anhui Province with Grant no. KJ2008B83ZC.

Open Access This article is distributed under the terms of the Creative Commons Attribution Noncommercial License which permits any noncommercial use, distribution, and reproduction in any medium, provided the original author(s) and source are credited.

\section{References}

1. Pezzé, L., Smerzi, A.: Phys. Rev. Lett. 102, 100401 (2009)

2. Helstrom, C.W.: Quantum Detection and Estimation Theory. Academic Press, New York (1976), Chap. VIII

3. Holevo, A.S.: Probabilistic and Statistical Aspect of Quantum Theory. North-Holland, Amsterdam (1982)

4. Wootters, W.K.: Phys. Rev. D 23, 357 (1981)

5. Braunstein, S.L., Caves, C.M.: Phys. Rev. Lett. 72, 3439 (1994)

6. Giovannetti, V., Lloyd, S., Maccone, L.: Science 306, 1330 (2004)

7. Luo, S.L.: Lett. Math. Phys. 53, 243 (2000)

8. Luo, S.L.: Proc. Am. Math. Soc. 132, 885 (2003)

9. Luo, S.: Phys. Rev. Lett. 91, 18 (2003)

10. Luo, S.L.: Chin. Phys. Lett. 12, 3127 (2006)

11. Luo, S.L.: Commun. Theor. Phys. 47, 597 (2007)

12. Invernizzi, C., Korbman, M., Venuti, L.C., Paris, M.G.A.: Phys. Rev. A 78, 042106 (2008)

13. Zanardi, P., Paris, M.G.A., Venuti, L.C.: Phys. Rev. A 78, 042105 (2008)

14. Kitagawa, M., Ueda, M.: Phys. Rev. A 47, 5138 (1993)

15. Wineland, D.J., Bollinger, J.J., Itano, W.M., Heinzen, D.J.: Phys. Rev. A 50, 67 (1994)

16. Law, C.K., Ng, H.T., Leung, P.T.: Phys. Rev. A 63, 055601 (2001)

17. Jin, G.R., Kim, S.W.: Phys. Rev. A 76, 043621 (2007)

18. Jin, G.R., Kim, S.W.: Phys. Rev. Lett. 99, 170405 (2007)

19. Jafarpour, M., Akhound, A.: Phys. Lett. A 372, 2374 (2007)

20. Li, Y., Castin, Y., Sinatra, A.: Phys. Rev. Lett. 100, 210401 (2008)

21. Yang, Y., Liu, W.F., Sun, Z., Wang, X.: Phys. Rev. A 79, 054104 (2009)

22. Ma, J., Wang, X.: Phys. Rev. A 80, 012318 (2009)

23. Søensen, A., Duan, L.M., Cirac, I., Zoller, P.: Nature 409, 63 (2001)

24. Ulam-Orgikh, D., Kitagawa, M.: Phys. Rev. A 64, 052106 (2001)

25. Messikh, A., Ficek, Z., Wahiddin, M.R.B.: Phys. Rev. A 68, 064301 (2003)

26. Wang, X., Sanders, B.C.: Phys. Rev. A 68, 012101 (2003)

27. Wang, X., Miranowicz, A., Liu, Y.X., Sun, C.P., Nori, F.: Phys. Rev. A 81, 022019 (2010)

28. Luati, A.: Ann. Stat. 32, 1770 (2004)

29. Yan, D., Wang, X., Wu, L.: Chin. Phys. Lett. 22, 521 (2005)

30. Yan, D., Wang, X., Song, L., Zong, Z.G.: Cent. Eur. J. Phys. 5, 367 (2007)

31. Liu, W.-F., Xiong, H.-N., Ma, J., Wang, X.: J. Theor. Phys. 49, 1073 (2010)

32. Lipkin, H.J., Meshkov, N., Glick, A.J.: Nucl. Phys. 62, 188 (1965)

33. Dusuel, S., Vidal, J.: Phys. Rev. B 71, 224420 (2005)

34. Vidal, J., Palacios, G., Mosseri, R.: Phys. Rev. A 69, 022107 (2004)

35. Barthel, T., Dusuel, S., Vidal, J.: Phys. Rev. Lett. 97, 220402 (2006)

36. Hill, S., Wootters, W.K.: Phys. Rev. Lett. 78, 5022 (1997)

37. Wootters, W.K.: Phys. Rev. Lett. 80, 2245 (1998)

38. Usha Devi, A.R., Wang, X., Sanders, B.C.: Quantum Inf. Process. 2, 207 (2003) 